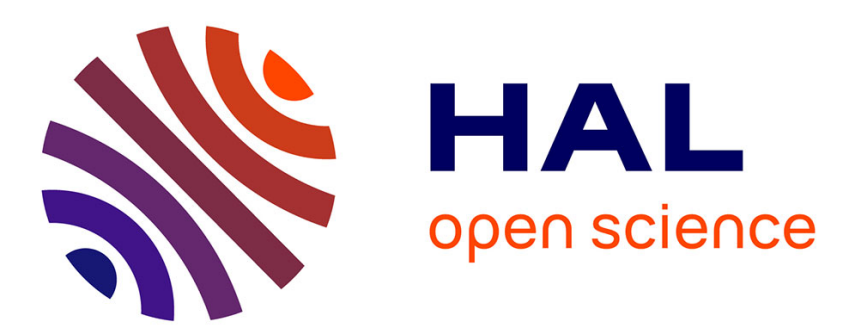

\title{
Pulsed laser determination of surface electric charge distributions
}

\author{
C. Alquié, G. Charpak, J. Lewiner
}

\section{To cite this version:}

C. Alquié, G. Charpak, J. Lewiner. Pulsed laser determination of surface electric charge distributions. Journal de Physique Lettres, 1982, 43 (19), pp.687-693. 10.1051/jphyslet:019820043019068700 . jpa00232112

\section{HAL Id: jpa-00232112 https://hal.science/jpa-00232112}

Submitted on 1 Jan 1982

HAL is a multi-disciplinary open access archive for the deposit and dissemination of scientific research documents, whether they are published or not. The documents may come from teaching and research institutions in France or abroad, or from public or private research centers.
L'archive ouverte pluridisciplinaire HAL, est destinée au dépôt et à la diffusion de documents scientifiques de niveau recherche, publiés ou non, émanant des établissements d'enseignement et de recherche français ou étrangers, des laboratoires publics ou privés. 


\title{
LE JOURNAL DE PHYSIQUE-LETTRES
}

J. Physique - LETTRES 43 (1982) L-687 - L-693

1er OCTOBRE 1982, PAGE L-687

Classification

Physics Abstracts

$06.70 \mathrm{D}-42.60-61.80 \mathrm{C}-87.60$

\section{Pulsed laser determination of surface electric charge distributions}

\author{
C. Alquié, G. Charpak (*) and J. Lewiner \\ Laboratoire d'Electricité Générale, Ecole Supérieure de Physique et de Chimie Industrielles, \\ 10, rue Vauquelin, 75005 Paris, France
}

(*) IN2P3, 20, rue Berbier du Mets, 75013 Paris, France.

\section{(Reçule 19 juillet 1982, acceptéle ler septembre 1982)}

\begin{abstract}
Résumé. - Nous proposons une méthode nouvelle de lecture, à haute résolution, d'une distribution de charges électriques à la surface d'un diélectrique. Cette méthode est basée sur la mesure de la variation des charges induites sur une sonde lorsque les charges à mesurer sont déplacées. Cette perturbation locale est réalisée à l'aide d'un laser pulsé focalisé sur une cible absorbante mince adjacente au diélectrique. En déplaçant le diélectrique par rapport au laser et à la sonde, la distribution peut être déterminée avec une résolution meilleure que $0,2 \mathrm{~mm}$.

Cette méthode pourrait ouvrir de nouvelles voies dans des domaines aussi variés que l'électrophotographie, le traitement d'image ou l'instrumentation médicale.
\end{abstract}

\begin{abstract}
We propose a new method for high resolution determination of surface electric charge distributions on a dielectric plate. The method is based on the measurement of the variation of the charges induced on a probe when the charges to be measured are being displaced. This local perturbation is achieved by means of a laser pulse focused on a thin absorbing target adjacent to the dielectric. By sweeping the laser beam and the probe parallel to the dielectric surface, the distribution can be determined with a resolution better than $0.2 \mathrm{~mm}$.

This method could open new approaches in various fields, such as electrophotography, image processing or medical instrumentation.
\end{abstract}

In various fields such as electrophotography, non destructive testing or medical imaging, the information to be processed can be obtained or stored as a distribution of electric charges on the surface of a dielectric sheet or plate. The visualization of this distribution is generally achieved using solid or liquid toners. It is clear however, that an electrical measurement of the density of surface charges, with a high spatial resolution would present many advantages since it is non destructive and opens the way to analog or digital signal analysis.

Several techniques have already been proposed in order to attain this goal [1-4]. They all involve the measurement of the charges induced on a probe by the electric field created by the charges to be measured. This can be done in various ways which require some modulation. For instance the distance between the probe and the dielectric plate is modulated (vibrating capacitor). In another configuration a vibrating or rotating vane is periodically inserted between the probe and the plate. Whatever the technique used, the charges on the probe are induced by charges distributed on an area $S$ of the plate larger than that of the probe, and which depends on geometrical parameters of the system. Such an effect is visible in figure $1 a$. In order to increase 
the resolution it is necessary to reduce not only the diameter of the probe, but also its distance to the dielectric plate, which creates new problems.

First it makes it mechanically difficult to sweep the surface of the plate. Second, the irregularities of the surfaces produce in these conditions large relative variations of this distance, which strongly affects the measurement.

In this paper we describe a new method which eliminates most of these problems and allows the determination of charge distributions with a higher resolution using a probe located at a larger distance from the plate. It is based on the fact that if a short duration perturbation is applied to a small area $s$ of the plate, it produces a local displacement of the charges of the plate and consequently a variation of the induction charge on the probe. Although the total charge $Q$ on the probe is induced by all the charges lying on $S$, the time dependent variation of $Q$ is only related to the charges which have been displaced, as shown in figure $1 b$.

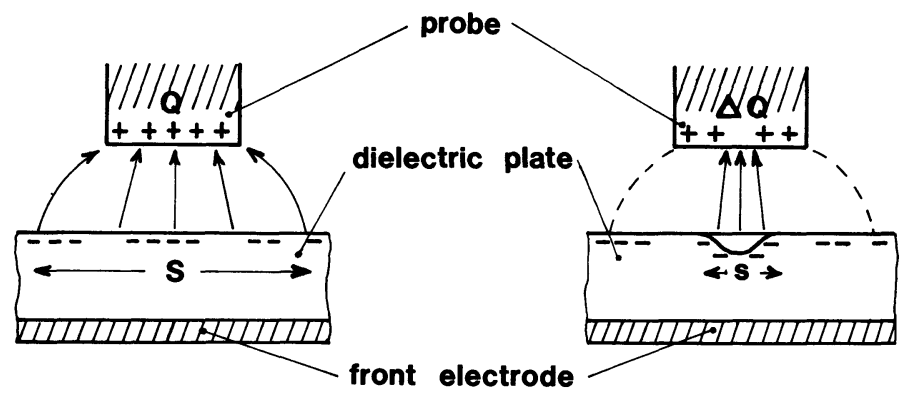

(a)

(b)

Fig. 1. - a) The total charge $Q$ on the probe is induced by charges distributed on an area $S ; b$ ) The variation $\Delta Q$ of the induction charge is related to the charges distributed on the small perturbed area $s$.

In order to produce such a perturbation, various techniques can be considared, involving acoustical, optical or electrical excitations of the local deformation. In the following, we describe an experiment using an optical excitation by a short duration laser pulse focused on a small area of the electrode opposite to the probe.

The generation of short-rise-time pressure waves by pulsed laser irradiation of metal targets has been intensively studied in the last ten years [5]. In various recent publications [6-9], this technique was used to determine the electric field or charge distributions inside dielectric materials. As was shown in reference [10], these distributions can be derived, using a one dimensional model, from the time variations of the open-circuit voltage or short-circuit current during the propagation of the pressure wave through the dielectric.

In the present paper, we use the impact of a short duration laser pulse focused on a metal target to produce a localized pressure wave which can be transmitted to the sample under investigation.

1. Theoretical. - We consider a dielectric plate of thickness $e$. One side is adjacent to an electrode; on the other side there is a non uniform distribution of surface charges $\sigma(x, y)$. We assume for simplicity that the dielectric plate has neither bulk space charges nor polarization. By focusing the laser beam at a point $\left(x_{0}, y_{0}\right)$ on the front electrode (Fig. 2), a local compressional wave is created and transmitted to the dielectric in which it travels at the sound velocity $v$. The related transit time is $\tau=e / v$. The main effect of this wave is to decrease locally the thickness of the dielectric plate of an amount $\Delta e$, and to change the dielectric constant in the compressed region. 
Assuming that the pressure wave is uniform on an area $s$ and null elsewhere, $\Delta e$ can be expressed as :

$$
\Delta e(t)=\chi \int_{z_{\mathbf{f}}}^{d_{0}} p(z, t) \mathrm{d} z
$$

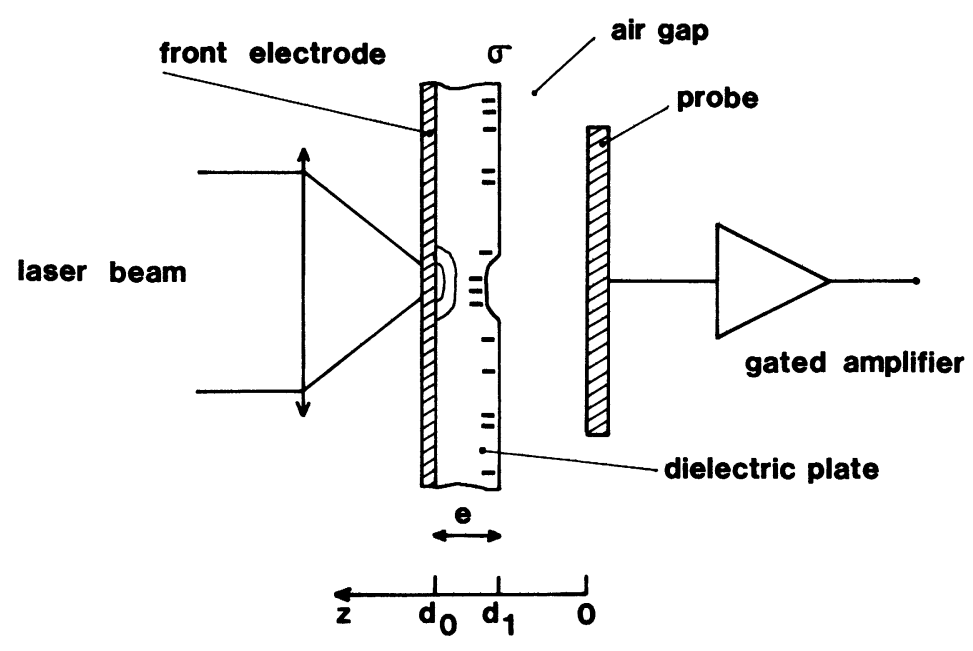

Fig. 2. - Schematic drawing of the experimental set-up.

$\chi$ is the compressibility of the material defined as $\chi=-V^{-1} \Delta V / \Delta p, z_{\mathrm{f}}=d_{0}-v t$ is the zero order expression of the position of the wave front $z_{\mathrm{f}}(t)$, and $p(z, t)$ is the pressure profile. In the case of a step function pressure wave of amplitude $\Delta p$, this expression reduces to $\Delta e(t)=\chi \Delta p v t$. The distance between the electrodes can be divided into three regions (see Fig. $3 b$ ), the first one related to the air gap $\left(0<z<d_{1}(t)\right)$, the second one to the uncompressed region of the dielectric plate $\left(d_{1}(t)<z<z_{\mathrm{f}}(t)\right)$ and the third one to the compressed region $\left(z_{\mathrm{f}}(t)<z<d_{0}(t)\right)$. In these regions, the permittivities are respectively $\varepsilon_{0}, \varepsilon=\varepsilon_{\mathrm{r}} \varepsilon_{0}$ and $\varepsilon^{\prime}=\varepsilon_{\mathrm{r}}^{\prime} \varepsilon_{0}$.

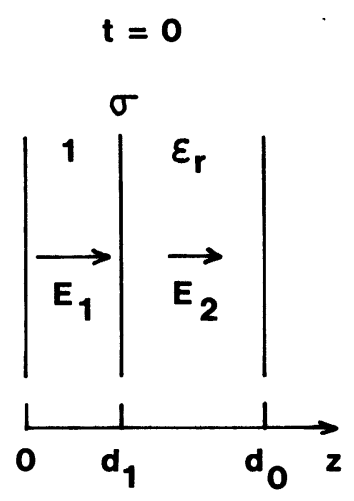

(a)

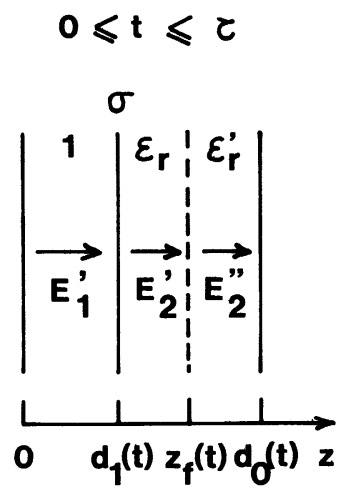

(b)

Fig. 3. - Configuration of the sample at the point $\left.\left(x_{0}, y_{0}\right): a\right)$ Before penetration of the compression wave; $b$ ) During the propagation of the wave front in the dielectric. 
A first order calculation can be made assuming that the diameter of the probe is large as compared to the lateral dimensions of the compressed region and to the distance between the probe and the front electrode. We assume also that in the compressed region the surface charge density is uniform $\sigma(x, y)=\sigma$. Since the electric field lines are constant in each region (no space charge), and using the continuity of the electrical displacement, the value of the charge $Q$ induced on the probe by the charges lying on $s$, before the propagation of the pressure wave in the sample, is, in short-circuit conditions :

$$
Q=-\sigma s e /\left(e+d_{1} \varepsilon_{\mathrm{r}}\right) .
$$

When $t<\tau$, the corresponding equilibrium value $Q^{\prime}(t)$ of the induction charge can be expressed as :

where

$$
Q^{\prime}(t)=-\sigma s e^{\prime}(t) /\left(e^{\prime}(t)+d_{1}(t) \varepsilon_{\mathrm{r}}\right)
$$

$$
e^{\prime}(t)=z_{\mathrm{f}}(t)-d_{1}(t)+\frac{\varepsilon_{\mathrm{r}}}{\varepsilon_{\mathrm{r}}^{\prime}}\left[d_{0}(t)-z_{\mathrm{f}}(t)\right]
$$

Consequently, if a deformation is produced on the dielectric plate the variation of the induction charge is $\Delta Q\left(x_{0}, y_{0}, t\right)=Q^{\prime}(t)-Q$. It must be noticed that $x_{0}$ and $y_{0}$ refer to the coordinates on the front electrode of the impact of the laser pulse. One has :

$$
\Delta Q\left(x_{0}, y_{0}, t\right)=-\sigma\left(x_{0}, y_{0}\right) s F(t, \Delta p) .
$$

For different pressure profiles, relation (2) still holds but a more complex function $F^{\prime}(t, p(z, t))$ has to be used instead of $F(t, \Delta p)$; this function can be derived using relation (1) and taking into account the pressure dependence of $\varepsilon_{\mathrm{r}}$.

Relation (2), which gives the time dependence of the signal produced by an impact at position $\left(x_{0}, y_{0}\right)$, makes it possible to obtain the spatial dependence of the signal at a given time $t_{0}$ after the impact of the laser pulse on the front electrode. It is proportional to the density of charge at the coordinates of the point of impact. We now assume that :

- the point of impact of the laser beam is kept along the axis of the probe,

- a lateral motion is imposed to the dielectric plate relative to this axis,

- the laser pulses, of duration $\tau_{0}$, are produced with a repetition rate $f_{R}=1 / T_{R}$, with $\tau_{0} \ll T_{\mathrm{R}}$ and $\tau \ll T_{\mathrm{R}}$.

In these conditions, the charge distribution on the plate can be directly derived from the successive measurements of $\Delta Q\left(x, y, N T_{\mathrm{R}}+t_{0}\right)$ where $N$ is an integer.

We can now apply the above calculation to the two following configurations.

a) The front electrode is rigid. This means that $d_{0}(t)=d_{0}, d_{1}(t)=d_{1}+\Delta e, z_{\mathrm{f}}(t)=d_{0}-v t+\Delta e$. To the first order in $\chi \Delta p$ one has :

$$
\Delta Q\left(x_{0}, y_{0}, t\right)=\sigma\left(x_{0}, y_{0}\right) s v t d_{1} \varepsilon_{\mathrm{r}}\left(e+d_{1} \varepsilon_{\mathrm{r}}\right)^{-2}\left[1-\frac{\varepsilon_{\mathrm{r}}}{\varepsilon_{\mathrm{r}}^{\prime}}(1-\chi \Delta p)+\chi \Delta p \frac{e}{d_{1}}\right] .
$$

In open-circuit experiments, the variation of the induction charge $\Delta Q(t)$ produces a voltage variation $V(t)=\Delta Q(t) /\left(C_{\mathrm{s}}+C_{\mathrm{s}}(t)\right)$, where $C_{\mathrm{s}}$ is the total capacity of the system, including stray capacities, and $C_{\mathrm{s}}(t)=\varepsilon_{0} \varepsilon_{\mathrm{r}} s /\left(e^{\prime}(t)+d_{1}(t) \varepsilon_{\mathrm{r}}\right)$ is the source capacity.

The main contribution to $C_{\mathrm{S}}$ will generally be related to the area $A$ of the probe, so that $C_{\mathrm{S}} \approx \varepsilon_{0} \varepsilon_{\mathrm{r}} A /\left(e+d_{1} \varepsilon_{\mathrm{r}}\right)$. Assuming that $A \gg s$, we obtain :

$$
V(t)=\frac{\Delta Q(t)}{C_{\mathrm{S}}}
$$


If a current amplifier is used the short-circuit current $I(t)=\mathrm{d}(\Delta Q) / \mathrm{d} t$ is measured.

$b$ ) The front electrode is a thin film evaporated on the dielectric. The compression produces a local variation of $d_{0}$, so that we can write $d_{0}(t)=d_{0}-\Delta e, d_{1}(t)=d_{1}, z_{\mathrm{f}}(t)=d_{0}-v t$. This leads to the same expression of $e^{\prime}(t)$ than in the previous case, and to the following first order in $\chi \Delta p$ expression of $\Delta Q(t)$ :

$$
\Delta Q\left(x_{0}, y_{0}, t\right)=\sigma\left(x_{0}, y_{0}\right) s v t d_{1} \varepsilon_{\mathrm{r}}\left(e+d_{1} \varepsilon_{\mathrm{r}}\right)^{-2}\left[1-\frac{\varepsilon_{\mathrm{r}}}{\varepsilon_{\mathrm{r}}^{\prime}}(1-\chi \Delta p)\right] .
$$

The only difference between equations (3) and (4) originates from the fact that in the first case $d_{1}$ depends on $t$, whereas in the second one $d_{1}$ remains constant.

2. Experimental and discussion. - In order to test the validity of the proposed method the following experimental set-up has been used. The pressure wave was produced by a $3 \mathrm{~ns} \mathrm{Nd}^{3+} / \mathrm{YAG}$ laser pulse which was focused on a $0.11 \mathrm{~mm}$ diameter spot at the surface of the target; the energy of the pulse was typically $10 \mathrm{~mJ}$. The target was made of a $15 \mu \mathrm{m}$ thick aluminum foil on which a $25 \mu \mathrm{m}$ thick F.E.P. teflon film was bonded. The opposite surface of the F.E.P. film was not metallized and was spaced $100 \mu \mathrm{m}$ away from the probe. Short-rise-time amplifiers (voltage or charge) were used in order to measure the open-circuit voltage or induced charge of the probe. After verifying that the uncharged film produced no signal, the sample was charged using an $8 \mathrm{keV}$ electron beam through a mask in which slots of $0.2 \mathrm{~mm}$ and $1.0 \mathrm{~mm}$ width had been cut.

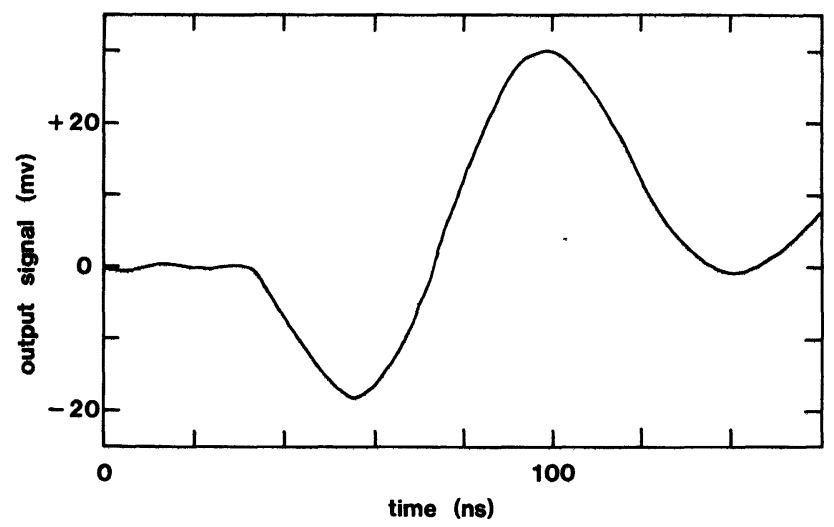

Fig. 4. - Variation of the induction charge versus time, during the propagation of the wave front in the dielectric. The sensitivity of the charge amplifier is $2 \mathrm{~V} / \mathrm{pC}$.

In figure 4, we show the output voltage of the charge amplifier as a function of time, for a shot in a charged region of the dielectric plate. The time scale originates when the laser pulse occurs. The flat part of the signal is associated with the propagation of the pressure pulse in the front electrode and to the delays introduced by the cables and the amplifiers. The transit time through the dielectric foil is $19 \mathrm{~ns}$. The sensitivity of the charge amplifier being $2 \mathrm{~V} / \mathrm{pC}$, one can obtain the total amount of charge in the perturbed region of the foil. It is of the order of $10^{-13} \mathrm{C}$.

Since in this single shot measurement the voltage signal to noise ratio is better than 10 , and can easily be improved by signal averaging the method allows detection of charge packages less than $10^{-15} \mathrm{C}$. 
It must be pointed out that the averaging is only possible in repetitive shots at the same location, provided that the signal does not change from one shot to the next one. This is only true with rather thick targets (front electrode), typically thicker than $10 \mu \mathrm{m}$. For lower thicknesses the signal amplitude in a single shot experiment is larger, but the front electrode is burnt out very rapidly.

The next step was to sweep the laser beam along the direction normal to the axis of the slots. Each measurement was made $18 \mathrm{~ns}$ after the penetration of the wave front in the dielectric. On figure 5, we show the distributions of the charges measured transverse to the slots.

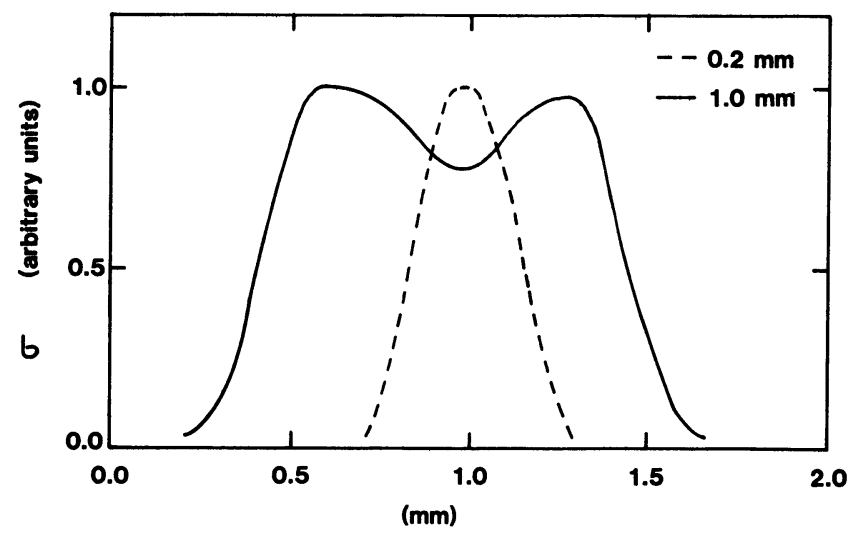

Fig. 5. - Distribution of the density of charges measured transversally to slots of $0.2 \mathrm{~mm}$ and $1.0 \mathrm{~mm}$.

The resolution obtained in these experiments, which is of the order of $0.2 \mathrm{~mm}$, can be improved by a sharper focusing of the laser beam.

It can be seen that depending on the relative dimensions of the focused beam diameter and of the front electrode thickness, the pressure wave which is transmitted to the dielectric may differ significantly from a locally plane wave (see Fig. 6). Nevertheless, as long as the lateral extent of the wave does not exceed the distance over which $\sigma$ changes significantly, the signal remains proportional to $\sigma(x, y)$, with a multiplying factor $F(t)$ which is a more complicated function of pressure and time. The spatial resolution of the method is limited either by the diameter of the beam, as illustrated on figure $6 a$, or by the thickness of the front electrode, as shown on figure $6 b$.

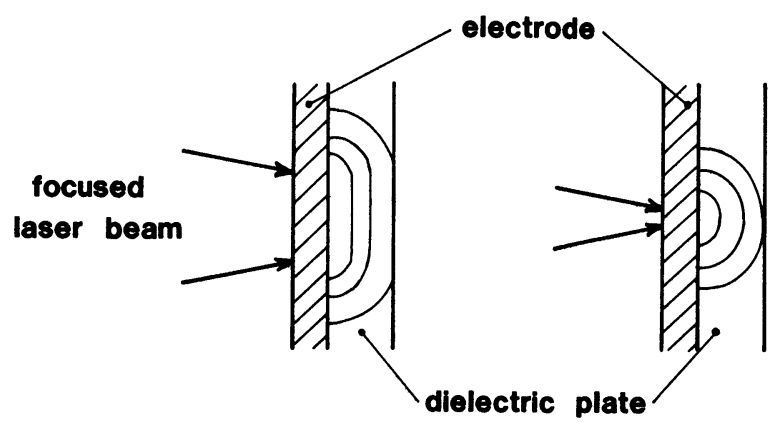

(a)

(b)

Fig. 6. - Shape of the wave front obtained if the front electrode thickness is : a) Smaller than the beam diameter; $b$ ) Larger than the beam diameter. 
Although the measurement can be made at any time $t<\tau$ following the penetration of the pressure pulse inside the dielectric, $t$ will be chosen to optimize the signal. If the pressure wave is a step function of time and if the measured quantity is either the open-circuit voltage or the variation of the induction charges on the probe, the signal increases linearly with time so that the measurement should occur just before the wave front reaches the charged surface. The same remark can be applied in the case of a pressure profile characterized by a short-rise-time and a width of the order of or longer than the transit time $e / v$. If the short-circuit current is measured, it will reach its maximum value at an instant of the order of the rise-time of the pressure wave.

In the case of a pressure pulse characterized by a duration $\tau_{\mathrm{p}}$ much shorter than $e / v$, the signal will be maximum when the pressure pulse has completely penetrated the dielectric, so that the measurement should be made at an instant $t$ of the order of $\tau_{\mathbf{p}}$.

The surface of the dielectric plate can be scanned using two different techniques : the laser beam and the probe can be moved simultaneously parallel to the dielectric, or the metallized dielectric can be moved between these two elements.

This new principle for measuring surface charges seems to be very attractive. It can be applied to situations where the charges are lying on the surface or at a rather constant distance from the surface. The mathematical analysis has been made assuming that the charges were localized at the surface. If one has a distribution of bulk charges, two cases can be considered : either the bulk charges are localized at a constant depth or they depend on the three coordinates. In the first case the above calculation can very easily be extended. In the second case one has to take into account the analysis described in references [7] and [10].

By improving the various above described parameters, it should be possible to reduce the power of each laser pulse. That would make easier the use of high repetition rate, short duration pulses, which would lead to rapid scanning of large areas of charged surfaces such as dielectric foils or photoconductors.

\section{References}

[1] Gross, B., Am. J. Phys. 12 (1944) 324.

[2] ReedyK, C. W., Perlman, M. M., J. Electrochem. Soc. 115 (1968) 49.

[3] Van Turnhout, J., Advances in Static Electricity, Vol. 1, ed. by W. de Gesst (Auxilia, Brussels) 1971, p. 56-81.

[4] For a review on these techniques, see : Topics in Applied Physics, Vol. 33, Electrets, ed. by G. M. Sessler (Springer-Verlag) 1980, p. 39-43.

[5] See, for example :

O,KeEFe, J. D., Skeen, C. H., Appl. Phys. Lett. 21 (1972) 464 ;

FoX, J. A., Barr, D. N., Appl. Opt. 12 (1973) 2547.

[6] Rozno, A. G., Gromov, V. V., Pis'ma Zh. Tekh. Fiz. 5 (1979) 648 (Sov. Tech. Phys. Lett. 5 (1979) 266).

[7] Alquí, C., Dreyfus, G., Lewiner, J., Phys. Rev. Lett. 47 (1981) 1483.

[8] Migliori, A., Hofler, T., Rev. Sci. Instrum. 53 (1982) 662.

[9] Sessler, G. M., West, J. E., Gerhard, R., Phys. Rev. Lett. 48 (1982) 563.

[10] Laurenceau, P., Dreyfus, G., Lewiner, J., Phys. Rev. Lett. 38 (1977) 46. 